\title{
Prevalencia de depresión posparto en puérperas adolescentes y adultas
}

\author{
Katherine L. Molero ${ }^{1}$, José Ramón Urdaneta Machado ${ }^{2}$, Charles Sanabria V. ${ }^{3}$, Nasser \\ Baabel Zambrano ${ }^{3}$, Alfi Contreras Benítez ${ }^{1}$, Estefany Azuaje Quiroz a , Nadia Baabel \\ Romero. a \\ ${ }^{1}$ División de Estudios para Graduados, ${ }^{2}$ Escuela de Bioanálisis. ${ }^{3}$ Facultad de Medicina, Escuela de Medicina. Universidad \\ del Zulia, Venezuela.
}

alumno. Escuela de Medicina, Universidad del Zulia. Venezuela.

\section{RESUMEN}

Objetivo: Comparar la prevalencia de depresión postparto (DPP) entre puérperas adolescentes y adultas atendidas en el Servicio de Obstetricia y Ginecología del Hospital "Dr. Manuel Noriega Trigo", en San Francisco, Estado Zulia, Venezuela. Métodos: Investigación comparativa con diseño no experimental, transeccional, de casos y controles, que incluyó una muestra de 100 mujeres en puerperio tardío, divididas en dos grupos pareados de 50 adolescentes (casos) y 50 adultas (controles); las que fueron evaluadas mediante la aplicación de la Escala de Depresión Postparto de Edimburgo (EDPS) y por un guión de entrevista para identificar los factores de riesgos asociados a esta patología. Resultados: Al comparar las puntuaciones totales en la EDPS las adolescentes mostraron un puntaje promedio menor que las adultas $(5,88 \pm 1,96$ vs. $11,58 \pm 2,9 ; p<0,001)$, mostrando significativamente una mayor frecuencia de de ansiedad $(p<0,05)$, sentimiento de culpa $(p<0,001)$ y dificultad para la concentración $(p<0,001)$, que es indicativo de un mayor riesgo de presentar DPP. Traduciéndose en $4 \%$ de puérperas adolescentes y $72 \%$ de las adultas con probable DPP, que al confirmarse mediante la entrevista del DSM IV mostró una prevalencia de $2 \%$ y $38 \%$, en adolescentes y adultas respectivamente. En las adultas predominó como factores de riesgo significativos el bajo nivel educativo $(p<0,05)$, las dificultades con la lactancia $(p<0,001)$ y la presencia de trastornos afectivos desde el embarazo $(p<0,001)$. Conclusión: La DPP fue más prevalente en las madres adultas que en las adolescentes.

\section{PALABRAS CLAVE: Adolescencia, depresión posparto, embarazo adolescente, puerperio}

\section{SUMMARY}

Aim: To compare the prevalence of postpartum depression in postpartum adolescents and adults treated in the Obstetrics and Gynecology Service at the Hospital "Dr. Manuel Noriega Trigo" in San Francisco, Zulia State, Venezuela. Methods: This is a comparative research with non-experimental and transectional design, which included a sample of 100 women in late postpartum, divided into two groups of 50 adolescents (cases) and 50 adults (controls), which were evaluated by application of Postpartum Depression Scale Edinburgh (EDPS) and an interview guide to identify risk factors associated with this condition. Results: Comparing the total scores in EDPS adolescent mothers showed lower average score than adults (5.88 \pm 1.96 vs. $11.58 \pm 2.9 ; p<0.001)$, who showing significantly greater frequency of anxiety $(p<0.05)$, guilt 
$(p<0.001)$ and impaired concentration $(p<0.001)$, which was indicative of an increased risk of postpartum depression in adults women. Resulting in $4 \%$ of and $72 \%$ of adolescents and adult postpartum women respectively, with postpartum depression likely that the interview confirmed by DSM IV showed a prevalence of $2 \%$ and $38 \%$, respectively adolescents and adults; predominantly in adult women as factors significant risk have a low educational level $(p<0.05)$, difficulties with breastfeeding $(p<0.001)$ and the presence of affective disorders from pregnancy $(p<0.001)$. Conclusion: The postpartum depression was more prevalent among the adult mothers.

\section{KEY WORDS: Adolescents, adolescent pregnancy, postpartum depression, puerperium}

\section{INTRODUCCIÓN}

Durante los últimos veinticinco años las conductas sexuales de hombres y mujeres se ha modificado como resultante de los cambios socioculturales, entre los cuales destaca la tendencia progresiva a la disminución de la edad de la primera relación sexual coital en adolescentes; cambios conductuales que constituyen una problemática de salud pública, ya que los exponen a riesgos tales como las enfermedades de transmisión sexual, embarazo precoz y aborto (1). El embarazo adolescente representa un importante problema de salud públi$\mathrm{ca}$, que incide directamente en la posibilidad de las jóvenes de completar a cabalidad las tareas de la adolescencia y de asumir los desafíos que implica el embarazo y el nacimiento de un hijo $(2,3)$.

En Venezuela, las autoridades sanitarias han asumido al embarazo en adolescentes como el segundo gran problema de salud sexual y reproductiva a nivel nacional, siendo el primero la alta cifra de mortalidad materna por causas prevenibles y ocupando el tercer lugar entre la causas de muerte en mujeres entre 15 y 19 años. El 50\% de las adolescentes venezolanas se inicia sexualmente antes de los 19 años y el $10 \%$ antes de los 15 , mientras que en áreas rurales la edad disminuye, el $30 \%$ se inicia antes de los 15 años y el $70 \%$ antes de los 19 (4).

Tomando en cuenta tanto que la mayor parte de las madres adolescentes pertenecen a los sectores sociales de recursos limitados, como que las circunstancias en que ellas crecen marcan de un modo peculiar su adolescencia, parece obvio que el resultado del embarazo dentro del contexto biológico y psicosocial no es nada prometedor, tanto para la madre como para el futuro niño. Entre las consecuencias que se originan del embarazo adolescente, se ha señalado que las mujeres adolescentes presentan más frecuentemente depresión postparto (DPP) que las mayores $(2,5)$, reportándose tasas de prevalencias dentro de este grupo etario de un 14 a $48 \%$ (6).
La DPP corresponde a una enfermedad del ánimo que se presenta frecuentemente en un período específico de la vida de la mujer, el puerperio (7). Otros la catalogan como un trastorno psíquico habitualmente recurrente (8). Moreno y cols, (9), le añaden a esta definición la ausencia de síntomas psicóticos y aunque el cuadro clínico que provoca es similar al de los episodios depresivos observados en otras etapas de la vida, posee características peculiares que justifican su consideración como entidad especifica. La DPP representa una importante afección de la salud mental en la mujer $(7,10-13)$, que en las madres adolescentes predice un mayor riesgo de deserción escolar, de abuso o negligencia hacia el niño y mayor probabilidad de un segundo embarazo adolescente (2).

Esta entidad constituye un problema de salud pública en varios países, con una prevalencia mundial que fluctúa entre un $10 \%$ a $15 \%$, tres veces más alta en los países en desarrollo que en los países desarrollados $(14,15)$, siendo mayor en las mujeres de nivel socioeconómico bajo $(9,13)$. En Latinoamérica se ha reportado una alta prevalencia de DPP: Chile 50,7\% (16), Colombia 32,8\% (17), México $32,6 \%$ (18), Perú $24,1 \%$ (19) y Venezuela $22 \%(12,13)$. Sin embargo, es difícil determinar la prevalencia real de este trastorno, porque los estudios difieren extremadamente en lo que se refiere a metodología empleada y a las poblaciones analizadas, pero puede aceptarse que 10 a $20 \%$ de las puérperas son afectadas por esta entidad (9).

El puerperio constituye una época en la cual los trastornos afectivos son comunes, pero algunos, como la DPP son poco reconocidos (20). Se sabe que durante el embarazo y el puerperio se suceden una serie de cambios bioquímicos, psicológicos y sociales que ocasionan una mayor vulnerabilidad para la aparición de trastornos en la esfera psíquica de la mujer $(13,14,21,22)$.

El método más adecuado para el diagnóstico parece ser el realizado en dos etapas: rastreo con un test de autoaplicación, seguido de entrevista 
diagnóstica en casos positivos (9). Para ello, se han utilizado varias escalas, siendo la escala para depresión del centro epidemiológico (CES-D) y la escala de Edinburgh para depresión posparto (EPDS), las más conocidas y usadas para la detección de la DPP (22). Otra escala cuya utilidad fue validada en poblaciones hispanas en Estados Unidos, es la de tamizaje para depresión posparto de Beck y Gable, la cual mostró ser útil para los posibles casos de DPP en esta población (23).

Considerándose tanto el embarazo adolescente como la DPP problemas de salud pública, es conveniente detectar estos casos en las puérperas adolescentes, para lo cual pudiesen aplicarse diversas técnicas de tamizaje que permitan identificar a aquellas madres con mayor necesidad de una evaluación diagnóstica más completa. Por tal motivo, esta investigación tuvo como objetivo comparar la frecuencia de DPP entre las mujeres adolescentes y adultas, atendidas en la consulta de orientación reproductiva del adolescente de la Unidad de Desarrollo Integral y Orientación Reproductiva del Adolescente (UDIORA), del Servicio de Ginecología y Obstetricia del Hospital "Dr. Manuel Noriega Trigo", en San Francisco, Estado Zulia, Venezuela.

\section{PACIENTES Y MÉTODOS}

Investigación de tipo comparativa con diseño no experimental, transeccional, de casos y controles, en la cual se seleccionaron 100 mujeres en etapa de puerperio tardío, separándolas en dos grupos pareados de 50 sujetos de acuerdo a si pertenecen al grupo adolescentes (casos) o adultas (controles). Las pacientes se parearon de acuerdo a su nivel socioeconómico y estado civil, procediéndose a aplicar un muestreo intencionado y no probabilístico.

Se incluyeron puérperas tardías, sin diagnóstico previo de enfermedades psiquiátricas, quienes manifestaron su deseo voluntario de participar en la investigación y suministraron consentimiento informado del mismo. Se excluyeron analfabetas, con discapacidad visual, incapacidad para comprender o responder preguntas, muertes perinatales, malformados, y recién nacidos enfermos o que se encontraban hospitalizados.

Como instrumento de evaluación se empleó, la escala de depresión postnatal de Edimburgo (EPDS), la cual es un instrumento de auto reporte de 10 ítems, que ha sido validada en múltiples países, demostrando tener un alto poder diagnóstico, con una sensibilidad cercana al $100 \%$ y una especificidad de $82 \%$ para evaluar la intensidad de la depresión postparto $(13,22,24,25)$; este instrumento fue diseñado específicamente como método de despistaje y no de diagnóstico para DPP (9). Cada ítems tiene cuatro opciones de respuesta con un puntaje que varía de 0 a 3 puntos, de acuerdo a la severidad de los síntomas, de modo que al final de la prueba, el puntaje varía entre 0 a 30 puntos. La escala mide la intensidad de los síntomas depresivos; 5 de estos ítems incluyen la disforia, 2 ítems están relacionados con ansiedad, 1 con sentimiento de culpa, 1 con ideas suicidas y 1 con dificultad de concentración (13). Según los resultados obtenidos la puérpera puede ser clasificada en tres grupos: (a) sin riesgo de depresión posparto $(<10$ puntos), (b) puntaje límite para riesgo de depresión (10-12 puntos), y (c) puntaje indicador de probable depresión ( $\geq 13$ puntos).

Se procedió a utilizar la técnica de la entrevista estructurada, resguardando la privacidad y confidencialidad de la paciente; para ello, se diseño un guión de entrevista, el cual estuvo conformado por cuatros secciones con quince instrucciones diseñadas para la identificación de los factores de riesgo asociados a DPP y un área para establecer la puntuación obtenida en la prueba tamiz y el resultado de la prueba confirmatoria.

Cada una de las pacientes seleccionadas fueron citadas para su valoración en una consulta posparto cuatro semanas después del parto, durante su puerperio tardío, para efectuar una evaluación médica integral en la cual mediante el interrogatorio clínico y con la ayuda del instrumento seleccionado se recogieron los datos que permitieron detectar la DPP en estas mujeres y cotejar la presencia o no de los factores de riesgo evaluados. A cada paciente se les informó sobre los propósitos de la investigación y previo consentimiento informado fueron finalmente incluidas en la misma. Importa destacar que el procedimiento a seguir en estas pacientes no representa un riesgo para salud de estas mujeres ni se violentan normas éticas; asimismo, dicho protocolo contó con la aprobación del Comité de Bioética del hospital y tuvo la aprobación tanto del comité de postgrado como de la subdirección de Docencia e Investigación de la Institución.

Durante la consulta postparto, a cada una de las puérperas se les registraron los siguientes datos: edad cronológica, paridad y tiempo de puerperio. Luego se les aplicó la EPDS, previa explicación de la misma. Las participantes completaron el cuestionario en un consultorio aislado para evitar el sesgo en las respuestas entregadas por interferencia de terceros o del propio personal adscrito al estudio. Seguidamente, se les efectuó una consulta médica integral, en donde se procedió a pesquisar los diferentes factores de riesgos mediante la entrevista clínica, para lo cual se siguió un guión de 
entrevista con el que se indagó acerca de factores de riesgo biológicos (trastornos médicos durante la gestación, enfermedad del neonato, operación cesárea, complicaciones del parto y dificultades con la lactancia) psicológicos (redes sociales limitadas, estrés, trastornos afectivos durante el embarazo como melancolía o depresión) y sociales (procedencia rural, vivienda precaria, bajo nivel educativo $y$ bajo ingreso familiar).

Las pacientes con una puntuación en la EDPS igual o mayor a 10 puntos, fueron catalogadas con una prueba tamiz positiva y se refirieron a la Consulta de Psiquiatría y Salud Mental, donde especialistas en esta área por medio de una entrevista semi-estructurada de acuerdo con el Manual de Diagnóstico y Estadísticas de Trastornos Mentales (DSM-IV) confirmaban la presencia o no de DPP.

Los datos obtenidos se organizaron en una base de datos y se empleó para su procesamiento el Paquete Estadístico para Ciencias Sociales (SPSS), versión 17; efectuándose un análisis estadístico de tipo descriptivo, expresándose mediante medidas de tendencia central: medias y desviación estándar $( \pm D E)$. Para realizar la comparación de los resultados obtenidos entre los dos grupos de pacientes evaluados, se utilizaron las pruebas de la T de Student para datos cuantitativos y la del Chi cuadrado para los datos cualitativos. Los resultados conseguidos fueron presentados en tablas de distribución de frecuencias.

\section{RESULTADOS}

En la Tabla I se muestra algunas características de las puérperas que participaron en el estudio. La edad promedio de las adolescentes fue de 16 años (rango: 14 y 18 años), la de las adultas de 28 años (rango: 24 y 32 años). Hubo menor paridad en las adolescentes en comparación con las adultas quienes eran multíparas en su mayoría. Igualmente se evaluó dentro de las características de la población, el tiempo posterior al parto para el momento que se realizó la evaluación, observándose menor tiempo de puerperio en el grupo de las adolescentes (promedio 20 días) en comparación con las adultas (22 días), no obstante, ambos grupos se encontraban en puerperio tardío.

En la Tabla II se presenta el análisis descriptivo de la EPDS, por ítems en puérperas adolescentes

\section{Tabla I CARACTERIZACIÓN DE LA MUESTRA}

\begin{tabular}{lrr}
\hline Análisis Descriptivo & $\begin{array}{c}\text { Adolescentes } \\
\text { Media } \pm \mathrm{DE}\end{array}$ & \multicolumn{1}{c}{$\begin{array}{c}\text { Adultas } \\
\text { Media } \pm \mathrm{DE}\end{array}$} \\
\hline Edad & $15,97 \pm 2,05$ & $27,6 \pm 4,25$ \\
Paridad & $1,35 \pm 0,72$ & $3,9 \pm 1,50$ \\
Tiempo de puerperio (días) & $19,95 \pm 10,20$ & $22,3 \pm 6,50$ \\
\hline
\end{tabular}

$\mathrm{n}=50$ para cada grupo

y adultas. Para el indicador disforia, las alternativas de respuestas mostraron una tendencia mayor a la alternativa 1 para los ítems 1 y 2 , evidenciándose en los resultados obtenidos para la mediana la cual alcanzó el valor de 1, es decir el $50 \%$ de las observaciones obtuvo un puntaje entre 1 y 2 , tal como se demuestra al observar el percentil 25 y 75 . Por otra parte, los ítems 7,8 y 9 que también medían al indicador disforia mostraron respuestas con tendencia a las alternativas 0 y 1 para el grupo de las adolescentes y madres adultas respectivamente. Es importante resaltar, que las alternativas favorables para la evaluación integral del test son las que se acercan a 0.

Para el indicador ansiedad, se observó que la alternativa considerada por ambos grupos para el ítem 4 fue la 2, mientras que para el ítem 5 la alternativa que presentó mayor tendencia fueron la 1 y 2 para adolescentes y adultas, respectivamente.

Para el indicador sentimiento de culpa, las alternativas que mostraron mayor tendencia en las respuestas fueron las alternativas 0 y 2 para las adolescentes y adultas, respectivamente.

Para el indicador dificultad de concentración, los resultados mostraron que para el ítem que lo mide, la respuesta con mayor frecuencia escogida por las adolescentes fue la número 1 , y para las adultas fue la 2 .

Finalmente, para el indicador ideación suicida, la alternativa que mostró una mayor tendencia fue la número 0 en ambos grupos de puérperas. En término generales, los resultados indican que las madres adolescentes muestran puntajes menores en la EPDS que las adultas, cuyos puntajes para todos los indicadores estudiados se ubicaron en valores mayores de 1. 


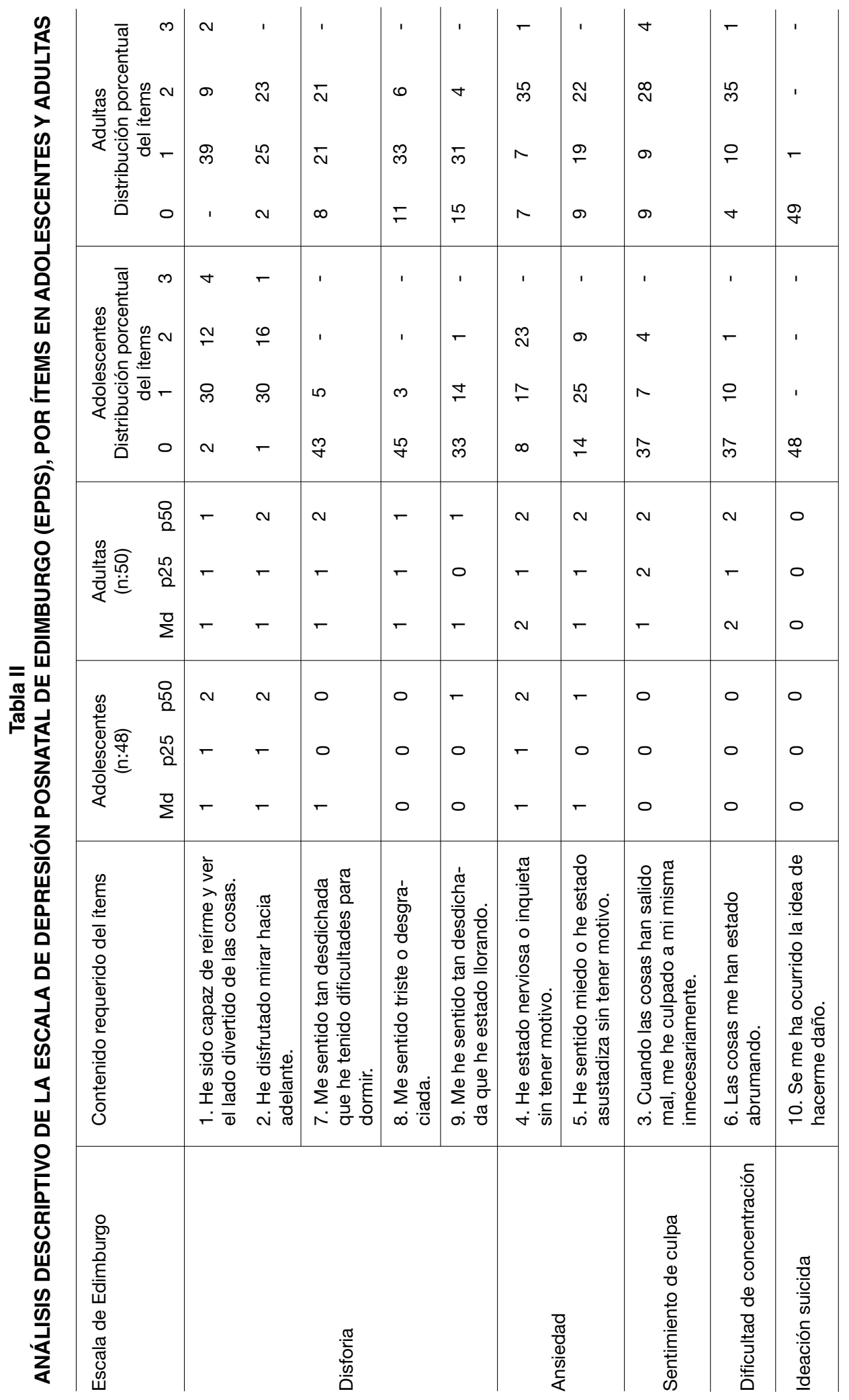


En la Tabla III se muestra la presencia de síntomas depresivos por la EPDS. Los resultados indican que las adolescentes presentaron menos síntomas depresivos que las adultas; en virtud que demostraron un menor porcentaje de ansiedad $(25,5 \%)$, sentimiento de culpa $(4,1 \%)$ y dificultad para la concentración $(1 \%)$ cuando se comparó con las puérperas adultas, quienes revelaron mayor frecuencia en todos estos síntomas depresivos (ansiedad 40,8\%; sentimiento de culpa 32,7\% y dificultad para la concentración $36,7 \%$ ); la significancia estadística que relacionó los indicadores ansiedad, sentimiento de culpa y dificultad para la concentración en ambos grupos evidenció diferencias significativas $(p<0,05)$. Junto a lo anterior, la disforia es un trastorno presente en ambos grupos; no obstante, aun cuando fue menor en el grupo de las adolescentes (12,2\%) en comparación con el grupo de mujeres adultas (13,3\%), esta diferencia no fue estadísticamente significativa $(p>0,05)$. Es importante mencionar, que ninguno de los grupos tuvo síntoma de ideación suicida.
En la Tabla IV se presenta el análisis descriptivo de la intensidad de los síntomas depresivos por la EPDS. Los resultados revelan que el $96 \%$ y el $28 \%$ de las madres adolescentes y adultas respectivamente, no mostraron riesgo de DPP, en virtud que, obtuvieron una frecuencia de puntaje menor a 10. Ninguna adolescente alcanzó un puntaje entre 10 y 12 , mientras que el $34 \%$ de las madres adultas si lo obtuvieron, presentando riesgo límite de DPP. De la misma forma, solo el $4 \%$ de las adolescentes obtuvo puntaje mayor de 13 , en tanto que la mayor frecuencia de las madres adultas fue clasificada en este grupo, lo que representa el $38 \%$ del total, puntaje que indica probable depresión. Al comparar los resultados de la puntuación obtenida en la EPDS, se evidenció que el grupo de adolescentes obtuvo un puntaje promedio de $5,88 \pm 1,96$, mientras que en las madres adultas fue de $11,58 \pm 2,9$ diferencia estadísticamente significativa $(p<0,05)$. El grupo de puérperas adultas al superar la puntuación de 10, indica nuevamente tendencia de riesgo a la DPP.

\section{Tabla III \\ SÍNTOMAS DEPRESIVOS SEGÚN LA ESCALA DE DEPRESIÓN POSNATAL DE EDIMBURGO (EPDS) EN ADOLESCENTES Y ADULTAS}

\begin{tabular}{lccccc}
\hline Síntomas Depresivos & \multicolumn{2}{c}{ Adolescentes } & \multicolumn{2}{c}{ Adultas } & \multicolumn{2}{c}{ Valor $\mathrm{p}^{*}$} \\
& $\mathrm{Fr}$ & $\%$ & $\mathrm{Fr}$ & $\%$ & $0,910^{\star *}$ \\
\hline Disforia & 12 & 12,2 & 13 & 13,3 & $0,003^{*}$ \\
Ansiedad & 25 & 25,5 & 40 & 40,8 & $0,000^{\star}$ \\
Sentimiento de culpa & 4 & 4,1 & 32 & 32,7 & $0,000^{\star}$ \\
Dificultad para la concentración & 1 & 1,0 & 36 & 36,7 & 0 \\
Ideación suicida & 0 & 0 & 0 & & - \\
\hline
\end{tabular}

* Prueba de Chi cuadrado. ** No significativo.

Tabla IV

\section{INTENSIDAD DE LOS SÍNTOMAS DEPRESIVOS POR LA ESCALA DE DEPRESIÓN POSNATAL DE} EDIMBURGO (EPDS) EN ADOLESCENTES Y ADULTAS

\begin{tabular}{llcccc}
\hline Intensidad de los síntomas depresivos & \multicolumn{2}{c}{ Adolescentes } & \multicolumn{2}{c}{ Adultas } & \multicolumn{2}{c}{ Valor $\mathrm{p}^{\star}$} \\
& Fr & $\%$ & Fr & $\%$ & - \\
Sin riesgo (<10 puntos) & 48 & 96,0 & 14 & 28,0 & - \\
Riesgo límite (10-12 puntos) & 0 & 0,0 & 17 & 34,0 & - \\
Probable depresión ( $\geq 13$ puntos) & 2 & 4,0 & 19 & 38,0 & - \\
Total & 50 & 100,0 & 50 & 100,0 & 0,000 \\
Puntuación EDPS & $5,88 \pm 1,96$ & & $11,58 \pm 2,9$ & \\
Media \pm DE & & & & \\
\hline
\end{tabular}

* Comparación de medias por T de Student. $\mathrm{P}<0,05$ valor significativo 
En la Tabla $V$ se presenta el análisis de riesgo de DPP por la EPDS. Los resultados indican que el $72 \%$ de las puérperas adultas presentaron pruebas de tamizaje positivo para riesgo de DPP y $28 \%$ resultaron negativas; mientras que las adolescentes tuvieron un riesgo de DPP del $4 \%$, resultando la mayoría de ellas negativas a la prueba tamiz (96\%). Al confirmar estos diagnósticos por medio del DSM IV, sólo 1 puérpera adolescente presentaba depresión menor (2\%), mientras que 19 adultas (38\%) presentaban tanto depresión menor $(n=16)$ como mayor $(n=3)$; como puede verse, al igual que lo determinado con la Escala de Edimburgo, las madres adultas presentaron un mayor diagnóstico de DPP.

En la Tabla VI se muestra la distribución de fre- cuencias y estimación de factores de riesgo para DPP en puérperas adolescentes y adultas. Los resultados ponen en evidencia que el principal factor de riesgo biológico para DPP fueron los trastornos médicos durante la gestación, y considerado como el factor de riesgo más importante para adolescentes $(49 \%)$ y adultas $(48 \%)$, seguido por la enfermedad del neonato (24,5\% y $30,6 \%$ respectivamente); no hubo diferencias estadísticamente significativa entre los grupos $(p>0,05)$ para ninguno de estos factores de riesgo. Cabe mencionar, que solo las dificultades con la lactancia materna fue el factor de riesgo biológico para la DPP que evidenció diferencia significativa $(p<0,05)$ entre los grupos $(25,5 \%$ vs. $3,1 \%$; adolescentes y adultas, respectivamente).

Tabla V

DIAGNÓSTICO DE DEPRESIÓN POSTPARTO SEGÚN ESCALA DE EDIMBURGOY DSM IV EN PUÉRPERAS ADOLESCENTES Y ADULTAS

\begin{tabular}{|c|c|c|c|c|c|c|c|c|}
\hline \multirow[b]{3}{*}{ Grupo } & \multicolumn{4}{|c|}{ Escala de Edimburgo } & \multicolumn{4}{|c|}{ DSM IV } \\
\hline & \multicolumn{2}{|c|}{+} & \multicolumn{2}{|c|}{-} & \multicolumn{2}{|c|}{+} & \multicolumn{2}{|c|}{-} \\
\hline & $\mathrm{Fr}$ & $\%$ & $\mathrm{Fr}$ & $\%$ & $\mathrm{Fr}$ & $\%$ & $\mathrm{Fr}$ & $\%$ \\
\hline Adolescentes & 2 & 4 & 48 & 96 & 1 & 2 & 49 & 98 \\
\hline Adultas & 36 & 72 & 14 & 28 & 19 & 38 & 31 & 62 \\
\hline
\end{tabular}

\section{Tabla VI \\ DISTRIBUCIÓN DE FRECUENCIA Y ESTIMACIÓN DE FACTORES DE RIESGOS PARA DEPRESIÓN POSTPARTO EN ADOLESCENTES Y ADULTAS}

\begin{tabular}{|c|c|c|c|c|c|}
\hline \multirow[t]{2}{*}{ Factor de riesgo } & \multicolumn{2}{|c|}{ Adolescentes } & \multicolumn{2}{|c|}{ Adultas } & \multirow[t]{2}{*}{ Valor $\mathrm{p}$} \\
\hline & $\mathrm{Fr}$ & $\%$ & $\mathrm{Fr}$ & $\%$ & \\
\hline \multicolumn{6}{|l|}{ Factores biológicos } \\
\hline Trastornos médicos en la gestación & 48 & 49,0 & 47 & 48 & 0,080 \\
\hline Enfermedad del neonato & 24 & 24,5 & 30 & 30,6 & 0,320 \\
\hline Operación cesárea & 12 & 12,2 & 15 & 15,3 & 0,580 \\
\hline Complicaciones del parto & 13 & 13,3 & 12 & 12,2 & 0,700 \\
\hline Dificultades con la lactancia materna & 25 & 25,5 & 3 & 3,1 & $0,000^{\star}$ \\
\hline \multicolumn{6}{|l|}{ Factores psicológicos } \\
\hline Trastornos afectivos en el embarazo & 40 & 40,8 & 17 & 17,3 & $0,000^{\star}$ \\
\hline Redes sociales limitadas & 27 & 27,6 & 36 & 36,7 & 0,100 \\
\hline Estrés & 38 & 39,2 & 36 & 37,1 & 0,510 \\
\hline \multicolumn{6}{|l|}{ Factores sociales } \\
\hline Procedencia rural & 16 & 16,4 & 9 & 9,2 & 0,080 \\
\hline Vivienda precaria & 10 & 10,2 & 14 & 14,3 & 0,410 \\
\hline Bajo nivel Educativo & 42 & 42,9 & 31 & 31,6 & $0,004^{\star}$ \\
\hline Bajo Ingreso familiar & 20 & 42,9 & 31 & 31,6 & 0,816 \\
\hline
\end{tabular}

\footnotetext{
* Prueba de Chi cuadrado, valor significativo.
} 
En este contexto, de acuerdo a los resultados obtenidos, el principal factor de riesgo psicológico para DPP de acuerdo a las puérperas adolescentes fueron los trastornos afectivos durante el embarazo $(40,8 \%)$ seguido por el estrés $(39,2 \%)$, mientras que para las adultas el principal factor psicológico fue el estrés $(37,1 \%)$ seguido por las redes sociales limitadas (36,7\%). De los factores de riesgo psicológico para DPP estudiados, el único que mostró diferencia significativa $(p<0,05)$ entre los grupos fueron los trastornos afectivos durante el embarazo, resultando que $40,8 \%$ de las adolescente frente a $17,3 \%$ de las adultas manifestaron la presencia de depresión o melancolía durante el transcurso de su gestación.

A lo anteriormente expuesto, el principal factor de riesgo social para DPP considerado para el grupo de adolescentes fue el bajo nivel educativo con $42,9 \%$, mientras que en adultas fue de $31,6 \%$, diferencia estadísticamente significativa $(p<0,05)$. Las puérperas adultas además consideran el bajo ingreso familiar $(31,6 \%)$ como uno de los factores de riesgo social para DPP, sin embargo no mostró diferencia significativa $(p>0,05)$ entre los grupos.

\section{DISCUSIÓN}

Los desórdenes afectivos ocurren frecuentemente durante el periodo posparto, la DPP puede ocurrir después de algún tiempo, pero más frecuentemente se presenta de la segunda a la tercera semana después del parto y puede durar hasta un año (26). Si bien el cuadro clínico es similar al de los episodios depresivos observados en otras etapas de la vida posee características peculiares que justifican su consideración como entidad específica $(9,13)$. Aunque se conoce que durante el puerperio tanto los cambios bioquímicos existentes así como el estrés pueden desencadenar estos trastornos (27), no hay evidencia de que un desbalance hormonal o de neurotransmisores sea la causa de la DPP, sin embargo, las teorías más acertadas hasta el momento son las relacionadas con los cambios hormonales y las susceptibilidades específicas de estas pacientes durante este periodo (23).

En cuanto a la edad como factor de riesgo existen discrepancias, algunos autores han señalado que las mujeres adolescentes presentaban más depresión que las mujeres mayores (6), representando el hecho de ser adolescente un factor de riesgo independiente para DPP (28). Al respecto una revisión de 40 artículos publicados entre 1990 y 2013 sugiere que las adolescentes embarazadas tenían un mayor riesgo de experimentar síntomas depresivos que las mujeres adultas embarazadas y puérperas (29).

Esta mayor prevalencia de DPP entre las madres adolescentes ha sido atribuida tanto a ciertos factores desmoralizadores en el entorno social como a experiencias pasadas, que tornan al adolescente más vulnerable a hechos vitales como el embarazo (5), como al antecedente de abuso físico y/o sexual en la infancia, lo cual se ha relacionado significativamente con la aparición de DPP entre las madres adolescentes (30), o al hecho de tener emociones negativas hacia el cuidado del bebé durante el embarazo (31). No obstante, el reporte de DPP en adolescentes muestra importantes fluctuaciones, con valores que oscilan entre 20 y $57 \%$ $(2,30,32-34)$.

Contrariamente, los resultados obtenidos en esta serie demostraron una mayor frecuencia de esta complicación en las puérperas en edad adulta; hallazgo similar a los encontrados por otros autores $(9,13,14,20)$, quienes han expresado que la edad no representa un factor de riesgo estadísticamente significativo para el hallazgo de test positivo para DPP, encontrándose que las madres adolescentes no presentan un riesgo mayor que el de otras mujeres para el desarrollo de esta entidad. Esta mayor frecuencia encontrada en las puérperas adultas podría explicarse por el hecho de que la prevalencia de depresión mayor aumenta con la edad, por lo que en las puérperas adultas pudiese ser mayor la frecuencia de esta patología, debido a que pudiese tratarse de recaídas de una depresión de base, desencadenada por los cambios hormonales productos del parto y los factores socio-culturales relacionados con la llegada de un nuevo hijo (20).

Asimismo, se ha sugerido que el número de partos es un factor asociado a la DPP, diversas investigaciones han concluido que el nacimiento del primer hijo representa un estrés único y se correlaciona con la depresión de manera más fuerte, que en el caso del segundo o el tercer parto (35). Sin embargo, al igual que los resultados obtenidos por otros autores $(9,10,20)$, en este estudio las adolescente estudiadas eran mayormente primigestas, por lo que la primiparidad no mostró ser un factor determinante para la presencia de DPP. Este hallazgo, es indicativo de que un instrumento de rastreo como el EDPS debería ser aplicado a todas las puérperas de forma universal y no restringirlo a poblaciones de supuesto mayor riesgo.

Los resultados presentados denotan que en las puérperas adultas prevaleció de forma significativa el sentimiento de culpa y la ansiedad al compararse con las adolescentes, en quienes predominó la 
ansiedad y la disforia como principales síntomas depresivos. Se debe tener en cuenta que los síntomas anímicos muchas veces se confunden con las características de este período adaptativo (36). En el caso de las jóvenes, los síntomas depresivos suelen acompañarse tanto de tristeza como de irritabilidad (37).

En cuanto a los factores de riesgo presente para el desarrollo de DPP predominaron de forma significativa entre las puérperas adultas el bajo nivel educativo, las dificultades con la lactancia o la presencia de trastornos afectivos desde el embarazo. Resultados que coinciden con los publicados en otro estudio (12) realizado en puérperas de la región donde se reportó que la presencia de trastornos médicos durante el embarazo, la enfermedad del neonato, no contar con el apoyo de su pareja, presentar antecedentes de depresión o melancolía, o tener un nivel educativo primario o inferior, mostraban una asociación significativa con el desarrollo de esta patología.

En cuanto a las dificultades en la lactancia, es conocido que los niveles de prolactina forman una curva inversa a la de los estrógenos y progesterona durante el periodo posparto, por lo que razonablemente se ha asumido que las madres que no amamantan a sus bebés y producen un rápido declive en su prolactina, podrían tener una mayor incidencia de DPP (35). Asimismo, otros autores (38-40) han señalado que tanto los problemas propios del posparto, como las dificultades en la lactancia o dar a luz un recién nacido de muy poco peso, como los antecedentes de depresiones previas, tendrían particular importancia en el desarrollo de esta entidad. De igual manera, Urdaneta y cols (12), encontraron que las mujeres con educación universitaria presenta significativamente un menor riesgo para el desarrollo de DPP; al igual que otros investigaciones que han reportado una asociación entre los niveles educativos más bajos con una mayor gravedad de la sintomatología depresiva $(15,18,38)$.

La Escala de Edimburgo ha resultado ser un buen método de pesquisa precoz de DPP, demostrando una alta precisión diagnóstica para el tamizaje de esta entidad en madres adolescentes (41). Los resultados de esta investigación demuestran un riesgo elevado de depresión, con pruebas de tamizaje positivo en $72 \%$ de las pacientes adultas y sólo 4\% entre las adolescentes; que al confirmarse con los resultados de la Encuesta Diagnóstica para Depresión según DSM-IV se obtuvo igualmente una mayor prevalencia en las puérperas adultas $(38 \% ; n=19)$ que en las adolescentes $(2 \% ; n=1)$, con una prevalencia global para toda la muestra estudiada del $20 \%(n=20)$; cifra similar a las encontradas por otros autores tanto en la literatura internacional como en la nacional $(9,10,13,42)$, lo cual hace patente el grave problema de subdiagnóstico observado en instituciones de asistencia maternoinfantil, principalmente en aquellas como la estudiada donde no se aplica ningún test de rastreo durante el período puerperal.

Tanto la presencia de la DPP en estas pacientes, como la influencia de los factores de riesgo biológicos, psicológicos y sociales, demuestra que la población estudiada presentaba una considerable prevalencia de esta entidad desde el punto de vista porcentual en función del valor total de la muestra, de allí la importancia de tenerla presente durante la evaluación prenatal, por el riesgo de que se presenten síntomas depresivos persistentes después de seis meses de posparto (23). No debe olvidarse que la salud mental materna durante los primeros meses de vida de su hijo es un componente primordial para el logro de un buen un vínculo madrehijo, donde se asentarán las bases de esta relación y la primera aproximación del niño a la vida (43), teniendo la DPP consecuencias negativas tanto a nivel del vínculo madre-hijo como en el desarrollo infantil, bien sea en términos emocionales, conductuales o cognitivos (44).

Es por ello, que los síntomas de malestar referidos por las mujeres embarazadas no deben ser subvalorados ni considerados como un componente normal de la experiencia del embarazo, puesto que el diagnóstico precoz y el tratamiento de la depresión durante la propia gestación revisten de una importancia significativa para evitarle a la mujer con depresión no tratada la aparición de la DPP. Prestar mayor atención a los síntomas de la depresión, puede mejorar la identificación de mujeres que sufren de depresión tanto en el embarazo como en el puerperio $(45,46)$. En este sentido, el uso de la EDPS permitió no sólo detectar un gran número de casos que suelen pasar desapercibidos, sino también identificar indirectamente otros factores estresantes para la madre, que se manifiesten en un mayor puntaje, y sobre los cuales es posible intervenir o brindar apoyo, como son la presencia de conflictos maritales, riesgo de violencia intrafamiliar o maltrato infantil $(8,25,47)$.

Una fortaleza de este estudio es que fue desarrollado en un centro de salud público, sistema que atiende a una considerable parte de la población, lo que permitió efectuar un seguimiento posparto mediante una consulta posnatal en una institución donde no se ha establecido un programa de salud para atender a la mujer posterior al parto, permitiéndole 
a las pacientes que fueron incluidas en la investigación, disfrutar de una continuidad de la atención hospitalaria y el acceso a especialistas, los cuales a su vez pudieron conocer y familiarizarse con el instrumento de pesquisa utilizado y participar en el diagnóstico y tratamiento de la DPP. Dentro de las limitaciones del estudio es que se incluyó un tamaño de muestreo relativamente pequeño, no obstante a pesar de no contar la institución en el ámbito de la investigación con una consulta postparto, se logró obtener una muestra, que si bien fue intencionada y no probabilística, estuvo representada por mujeres con características demográficas diferentes que permitió evaluar los diferentes factores de riesgo estudiado para la estimación de padecer DPP.

\section{CONCLUSIÓN}

Al comparar las puntuaciones totales en la EDPS las madres adolescentes mostraron un puntaje promedio menor que las adultas $(p<0,001)$, indicativo de un menor riesgo de presentar DPP, realizándose el diagnóstico presuntivo de esta entidad de $4 \%$ en puérperas adolescentes y $72 \%$ de las adultas, que al confirmarse mediante la entrevista del DSM IV determinó una prevalencia de $2 \%$ y $38 \%$, respectivamente. En términos generales, se encontró una prevalencia de puérperas deprimidas del $20 \%$, cifra sorprendentemente alta para un país como Venezuela donde existe un subregistro de esta patología, puesto que no se pesquisa de forma sistemática. Por tanto, se recomienda incluir dentro de la evaluación posnatal la aplicación de la Escala de Edimburgo, con la finalidad de detectar oportunamente síntomas depresivos y diagnosticar oportunamente esta patología, para evitar sus consecuencias en la madre como y los recién nacidos.

\section{REFERENCIAS}

1. Fétis G, Bustos L, Lanas F, Baeza B, Contreras J, Hebel E, Marucich C. Factores asociados al uso de anticonceptivos en estudiantes de enseñanza media de la comuna de Temuco. Rev Chil Obstet Ginecol 2008;73(6):362-9.

2. Wolff C, Valenzuela P, Esteffan K, Zapata D. Depresión posparto en el embarazo adolescente: análisis del problema y sus consecuencias. Rev Chil Obstet Ginecol 2009;74(3):151-8

3. Gallo NE. Línea de base del proyecto de salud sexual y reproductiva para adolescentes en Medellín: componente cualitativo. Rev Fac Nac Salud Pública 2009;27 (3):282-90.

4. Organización Mundial de la Salud. Análisis preliminar de la situación de salud de Venezuela. Gobierno y Población: El desafió para la salud y el desarrollo. [Docu- mento en línea] Disponible en: http://www.OMS-OPS. com. Fecha de recuperación: 15 de Diciembre de 2011.

5. Bulnes MS, Ponce CR, Huerta RE, Elizalde R, Santiváñez WR, Aliaga JR; Álvarez L, Calmet R. Ajuste psicosocial y estado depresivo en adolescentes de centros escolares de Lima Metropolitana. Rev Invest Psicol 2005;8(2):23-39.

6. Kabir K, Sheeder J, Stevens C. Depression, weight gain, and low birth weight adolescent delivery: do somatic symptoms strengthen or weaken the relationship? J Pediatr Adolesc Gynecol 2008;21:335-42.

7. Rojas G, Fritsch R, Guajardo V, Rojas F, Barroilhet S, Jadresic E. Caracterización de madres deprimidas en el posparto. Rev Med Chile 2010;138(5):536-42.

8. Escobar J, Pacora P, Custodio N, Villar W. Depresión posparto: ¿se encuentra asociada a la violencia basada en género? An Fac Med 2009;70(2):115-8.

9. Moreno A, Domíngues L, França PS. Depresión posparto: prevalencia de test de rastreo positivo en puérperas del Hospital Universitario de Brasilia, Brasil. Rev Chil Obstet Ginecol 2004;69(3):209-13.

10. Urdaneta JR, Rivera AT, García J, Guerra M, Baabel NS, Contreras AJ. Prevalencia de depresión posparto en primigestas y multíparas valoradas por la Escala de Edimburgo. Rev Chil Obstet Ginecol 2010;75(5):31220.

11. Bowen A, Muhajarine N. Prevalence of antenatal depression in women enrolled in an outreach program in Canada. J Obstet Gynecol Neonatal Nurs 2006;35(4):49-8.

12. Urdaneta JR, Rivera AT, García J, Guerra M, Baabel NS, Contreras AJ. Factores de riesgo de depresión posparto en puérperas venezolanas valoradas por medio de la escala de Edimburgo. Rev Chil Obstet Ginecol 2011;76(2):102-12.

13. Pérez R, Saez K, Alarcon L, Aviles V, Braganza I, Coleman J. Variables posiblemente asociadas a depresión posparto, según escala Edimburgo. Rev Obstet Ginecol Venez 2007;67(3):187-91.

14. Evans G, Vicuña M, Marín R. Depresión posparto realidad en el sistema público de atención de salud. Rev Chil Obstet Ginecol 2003;68(6):491-4.

15. Alvarado R, Rojas M, Monardes J, Perucca E, Neves $E$, Olea E, Vera A. Cuadros depresivos en el posparto en una cohorte de embarazadas: construcción de un modelo causal. Rev Chil Neuro-Psiquiat 2000;38(2):84-93.

16. Póo AM, Espejo C, Godoy C, Gualda M, Hernández $\mathrm{T}$, Pérez C. Prevalencia y factores de riesgo asociados a la depresión posparto en puérperas de un Centro de Atención Primaria en el Sur de Chile. Rev Méd Chile 2008;136(1):44-52.

17. Bonilla OA. Depresión y factores asociados en mujeres adolescentes embarazadas y no embarazadas en Medellín (Colombia) 2009. Estudio de corte transversal. Rev Col Obstet Ginecol 2010;61(3):207-13.

18. Alvarado $C$, Cifuentes $A$, Estrada $S$, Salas $C$, Hernández AB, Ortiz SG, García CR, Torres A, Sandoval F. Prevalencia de depresión posnatal en mujeres atendidas en hospitales públicos de Durango, México. Gac Méd Méx 2010;146(1):1-9. 
19. Aramburú $P$, Arellano R, Jáuregui $S$, Pari L, Salazar $P$, Sierra O. Prevalencia y factores asociados a depresión posparto en mujeres atendidas en establecimientos de salud del primer nivel de atención en Lima Metropolitana, junio 2004. Rev Peru Epidemiol 2008;12 (3):1-5.

20. Latorre JF, Contreras LM, García S, Medina JA. La Depresión postparto en madres adolescentes de Bucaramanga, Colombia. Rev Col Obstet Ginecol 2006;57(3):156 -162.

21. Marchesi C, Bertoni S, Maggini C. Major and minor depression in pregnancy. Obstet Gynecol 2009;113:1292-8.

22. Campo A, Ayola C, Peinado HM, Amor M, Cogollo Z. Escala de Edinburgh para depresión postparto: consistencia interna y estructura factorial en mujeres embarazadas de Cartagena, Colombia. Rev Colomb Obstet Ginecol 2007;58(4):277-83.

23. Orejarena SJ. Trastornos afectivos posparto. MedUNAB 2004;7(20):134-9.

24. Oquendo M, Lartigue T, González I, Méndez S. Validez y seguridad de la Escala de Depresión Perinatal de Edinburgh como prueba de tamiz para detectar depresión perinatal. Perinatol Reprod Hum 2008;22(3):195-202

25. Castañón C, Pinto J. Mejorando la pesquisa de depresión posparto a través de un instrumento de tamizaje, la escala de depresión posparto de Edimburgo. Rev Méd Chile 2008;136(7):851-8.

26. Morris JK, Comerford M, Bernstein PS. Screening for postpartum depression in an inner-city population. Am J Obstet Gynecol;2003(188):1217-9.

27. Alonso SJ, Damas C, Navarro E. Behavioral despair in mice after prenatal stress. J Physiol Biochem 2000;56(2): 77-82.

28. Figueiredo B, Pacheco A, Costa R. Depression during pregnancy and the postpartum period in adolescent and adult Portuguese mothers. Arch Womens Ment Health 2007;10(3):103-9.

29. Siegel RS, Brandon AR. Adolescents, Pregnancy, and Mental Health. J Pediatr Adolesc Gynecol 2014;27(3):138-50.

30. Lesser J, Koniak-Griffin D. The impact of physical abuse on chronic depression in adolescent mothers. $J$ Pediatr Nurs 2000;15(6):378-87.

31. Secco M, Profit S, Kennedy E, Walsh A, Letorneau $\mathrm{N}$, Steward M. Factors affecting postpartum depressive symptoms of adolescent mothers. JOGNN 2007;36(1):47-54

32. Logsdon $M$, Hertweck $P$, Ziegler C, Pinto-Foltz. Testing a bioecological model to examine social support in postpartum adolescents. J Nurs Scholarsh 2008;40(2):116-23.
33. Cox J, Buman M, Valenzuela J, Pierre JN, Mitchell A, Woods E. Depression, parenting attribute, and social support among adolescent mothers attending a teen tot program. J Pediatr Adolesc Gynecol 2008;21(5):275-81.

34. Schmidt R, Wiemann C, Rickert V, Smith E. Moderate to severe depressive symptoms among adolescent mothers followed four years postpartum. J Adolesc Health 2006;38(6):712-8.

35. Vargas JE, García M. Depresión post-parto: presencia y manejo psicológico. Centro Regional de Investigación en Psicología 2009;3(1):11-18.

36. Dois A, Uribe C, Villarroel L, Contreras A. Factores de riesgo asociados a síntomas depresivos post parto en mujeres de bajo riesgo obstétrico atendidas en el sistema público. Rev Med Chile 2012;140(6):719-25.

37. Shanok A, Miller L. Depression and treatment with inner city pregnant and parenting teens. Arch Womens Ment Health 2007;10 (5):199-210.

38. Rojas G, Fritsch R, Solís J, González M, Guajardo V, Araya $R$. Calidad de vida de mujeres deprimidas en el posparto. Rev Méd Chile 2006;134(6):713-20.

39. Sierra JM, Carro T, Ladrón E. Variables asociadas al riesgo de depresión posparto. Edinburgh Postnatal Depression Scale. Aten Primaria 2002;30(2):103-11.

40. Steiner M. Perinatal mood disorders: position paper. Psychopharmacol Bull 2008;34:301-6.

41. Venkatesh KK, Zlotnick C, Triche EW, Ware C, Phipps MG. Accuracy of brief screening tools for identifying postpartum depression among adolescent mothers. Pediatrics 2014;133(1):e45-53.

42. Gjerdingen D, Crow S, McGovern P, Miner M, Center B. Postpartum depression screening at well-child visits: validity of a 2-questions screen and the PHQ-9. Ann Fam Med 2009;7(1):63-70.

43. Sheeder J, Kabir K, Stafford B. Screening for postpartum depression at well-child visits: is once enough during the first 6 months of life? Pediatrics 2009;123(6);e982-e988.

44. Jadresic E. Depresión en el embarazo y el puerperio. Rev Chil Neuro-Psiquiat 2010;48(4):269-78

45. Nardi B, Bellantuono C, Moltedo A. Eficacia de la terapia cognitivo conductual en la prevención de la depresión posparto. Rev Chil Obstet Ginecol 2012;77(6):434-43

46. Dennis CL, Ross LE. The clinical utility of maternal self-reported personal and familial psychiatric history in identifying women at risk for postpartum depression. Acta Obstet Gynecol Scand 2006;85:1179-85.

47. Canaval GE, González MC, Martínez L, Tovar MC, Valencia C. Depresión postparto, apoyo social y calidad de vida en mujeres de Cali, Colombia. Colombia Méd 2000;31(1):4-10. 